\title{
Computational Structural Engineering: Past Achievements and Future Challenges
}

\author{
Vagelis Plevris $^{1 *}$ and George C. Tsiatas ${ }^{2}$ \\ ${ }^{1}$ Department of Civil Engineering and Energy Technology, OsloMet-Oslo Metropolitan University, Oslo, Norway, \\ ${ }^{2}$ Department of Mathematics, University of Patras, Rio, Greece
}

Keywords: computational methods, structural engineering, challenges, FEM, BEM, meshless methods

\section{PAST ACHIEVEMENTS AND CURRENT TRENDS}

Computational methods are computer-based methods used to numerically solve mathematical models that describe physical phenomena. The purpose of computational modeling is to study the behavior of complex systems by means of computer simulations and it can be used to make predictions of the system's behavior under different conditions, often for cases in which intuitive analytical solutions are not available (Nature, 2018). Computational methods have emerged in engineering during the 1960s. Since then, structural engineers have been leaders in technological solutions to engineering analysis and design problems. The evolution of electronic computers together with the tremendous increase of computational power has triggered the continuous development of computational methods. Rapid advances in computer hardware have had a profound effect on various engineering disciplines. The applications are numerous and cover a broad field of engineering branches including civil, mechanical, naval, electrical, aerospace, material, biomolecular, among others. Computational structural engineering has evolved as an insightful blend combining both structural analysis and computer science.

Among all computational methods, the Finite Element Method (FEM) and the Boundary

OPEN ACCESS

Edited and reviewed by: Izuru Takewaki,

Kyoto University, Japan

*Correspondence: Vagelis Plevris vageli@oslomet.no

Specialty section:

This article was submitted to Computational Methods in Structural Engineering,

a section of the journal Frontiers in Built Environment

Received: 09 March 2018 Accepted: 23 March 2018

Published: 10 April 2018

Citation:

Plevris V and Tsiatas GC (2018) Computational Structural Engineering:

Past Achievements and Future Challenges. Front. Built Environ. 4:21. doi: 10.3389/fbuil.2018.00021
Element Method (BEM) are the most prevalent ones. Both methods exhibit unique characteristics as well as advantages and disadvantages. The FEM, as a simulation tool, enables sophisticated methods of computational mechanics, computer technology and applied mathematics. It has been broadly adopted in scientific research and engineering applications and it can be considered as the most popular method used for structural analysis, tackling linear and nonlinear problems of systems with various geometries, material properties and loads (Reddy, 2005). In FEM, the solution domain is subdivided into a finite number of elements. Each element approximately reproduces the behavior of a small region of the body it represents, but continuity between the elements is only enforced in an overall minimum energy sense. The FEM is especially suitable for problems with complex geometries, but the whole-body discretization scheme that is utilized inevitably leads to a large number of finite elements and, thus, increased computational cost. Although Professor R. Clough coined the term "Finite Element Method" in his pioneer work dated back in 1960 (Clough, 1960), the answer to the question "who invented FEM in everyday use?" is possibly M. Jonathan (Jon) Turner at Boeing who generalized and perfected the Direct Stiffness Method, and convinced his company to commit resources to it, over the period 1952-1964 (Felippa, 2017). The FEM obtained its real impetus in the 1960s and 1970s by the developments of pioneers J. H. Argyris, R. W. Clough, H. C. Martin, O. C. Zienkiewicz and their co-workers who evolved the method and applied it to a wide range of structural problems and applications.

In the years since its first use, FEM has grown and developed into a standard in design engineering worldwide. While the applications and technological capabilities may vary between different software programs, the cornerstone principle of the methodology remains the same. Although significant developments have been made in FEM over the past decades, there are still 
many technical challenges that remain outstanding, while new challenging problems keep emerging with the growth of explorations in science, engineering and technology. New novel principles, techniques, algorithms and methods are continuously being developed to improve the precision, speed, robustness and applicability of FEM.

On the other hand, BEM has proven to be an alternative to FEM computational method which offers different computational potentialities. In BEM the governing differential equations are initially transformed into equivalent integral ones, which in turn are discretized on the boundary of the solution domain. In this respect the dimension of the problem is reduced by one order and the number of unknowns is significantly reduced as well. Additionally, BEM allows the evaluation of the derivatives of the solution at any point of the domain of the problem, whereas it is suitable for the analysis of structures with complex boundaries and geometric peculiarities. In 1903, Fredholm was the first scholar who employed singular boundary integral equations (Fredholm, 1903), as a mathematical tool, to calculate the unknown boundary quantities for potential problems. Since at that time closed form solutions could only be derived for simple geometries, the method was practically ignored until the beginning of electronic computers. It was not until the early 1960s when Jaswon (1963) and Symm (1963) used Fredholm's equations to solve some two-dimensional problems of potential theory (Katsikadelis, 2016). A special reference should be made to pioneer Prof. Carlos Brebbia, renowned throughout the world as the originator of the BEM, who passed away only recently. He wrote one of the first books in BEM (Brebbia, 1980) and organized the first international conference on Recent Advances in BEM, back in 1978.

In the following years the development of the two computational methods was expeditious with numerous applications in engineering practice including: static and dynamic analysis of beams, plates, shells and membranes; linear and nonlinear problems of elasticity; orthotropic, anisotropic, composite and layered materials; plasticity; viscoelasticity; earthquake engineering; continuum mechanics; fracture mechanics and geomechanics; soil-structure engineering; size and shape optimization; to name only a few.

In the past two decades, meshfree or mesh reduction methods have been developed gaining tremendous traction among scientists, researchers and engineers. Trying to alleviate mainly the tedious meshing of the traditional element methods (especially FEM), meshfree methods do not require connection between nodes and the approximation of unknowns in the partial differential equations (PDEs) is constructed based on scattered points, within the problem domain and on its boundaries, without mesh connectivity (Chen et al., 2017). The earliest development of a meshfree method was the Smoothed Particle Hydrodynamics (SPH) introduced by Gingold and Monaghan (1977) and Lucy (1977) initially for astrophysical problems, while for solid mechanics the method has been applied by Libersky et al. (1993). The method exhibited many inaccuracies especially near boundaries and tension instabilities.

Many and various meshfree methods have emerged in the literature over the years, which mainly fall into two major categories based on the problem formulation, weak or strong. All the Galerkin meshfree methods belong in the weak form category (see e.g., Liu et al., 1995; Babuška and Melenk, 1997) being confronted with domain integration, and special treatment of the essential boundary conditions. On the other hand, the collocation meshfree methods (see e.g., Kansa, 1990a,b; Chi et al., 2013) are classified in the strong form category, suffering from very ill-conditioned systems. For more information on meshfree methods, the interested reader can consult the excellent review paper by Chen et al. (2017).

Similarly, mesh reduction methods use numerical techniques to reduce the domain mesh size or to reduce the discretization over the boundary of the body. For example, in BEM the presence of body forces generates domain integrals that can be computed by domain discretization. This, however, spoils the pure boundary character of the method. Several BEM-based methods have been reported in the literature which overcome the problem of domain integrals evaluation. Among them the Dual Reciprocity Method (DRM) (Partridge et al., 1991) and the Analog Equation Method (AEM) (Katsikadelis, 1994) are actually the most competent ones. Both methods can maintain the pure boundary character since discretization and integration are limited only on the boundary.

Other interesting and promising methods have also been proposed and developed lately. Isogeometric analysis (IGA) represents a recently developed technology in computational mechanics that offers the possibility of integrating FEA into conventional NURBS-based CAD design tools (Hughes et al., 2005). On the other hand, the extended finite element method (XFEM) is a numerical technique which extends the classical FEM approach by enriching the solution space for solutions to differential equations with discontinuous functions (Moës et al., 1999). Based on the generalized FEM and the partition of unity method (PUM), the method was developed to ease difficulties in solving problems with localized features (e.g., discontinuities) that are not efficiently resolved by mesh refinement.

There are numerous software packages available today for the simulation and analysis of structures, either commercial, free to use, or completely open-source. The philosophy of open source software is that it is developed by a community, in a collaborative manner, producing a reliable, high quality software quickly and inexpensively. An excellent such example of open-source FEM software is the "Open System for Earthquake Engineering Simulation" (OpenSees) (Mazzoni et al., 2006). It is an objectoriented software framework created at the National Science Foundation (NSF)-sponsored Pacific Earthquake Engineering (PEER) Center, mostly used for the FE simulation of the response of structures subjected to earthquakes.

\section{FUTURE CHALLENGES IN COMPUTATIONAL METHODS}

Given the development of computational methods and relevant tools during the last decades, very powerful capabilities are available today for the simulation and analysis of structures 
(Bathe, 2003). Nevertheless, there are still many exciting research challenges. Some of the future challenges are summarized below.

\section{System Identification and Modeling of Physical Systems}

The foremost challenge in structural engineering and, in general, computational mechanics is two-fold. First, the actual modeling of complex physical phenomena and the derivation of the equations governing their response; second, the development of the necessary computational tools that can accurately solve the respective equations. To these directions System Identification and Fractional Calculus play a dominant role. System identification is the process of modeling an unknown physical system based on a set of known input-outputs values (Sirca and Adeli, 2012). In order to identify the constitutive laws of the material or the degree of nonlinearity of the problem, fractional derivatives of constant or variable order (Katsikadelis, 2018) must be employed. Moreover, Variable Order (VO)Calculus, besides the suitable modeling of actual structures, may model the nonlinear response of constant order differential equations as linear response in a VO-Calculus framework, with all the simplifications that arise from the use of linear operators (Coimbra, 2003).

\section{Multi-Scale Modeling}

Numerical analysis and simulation at the nano-scale is a major challenge that will open up a huge field in the future. This can lead to applications in biological engineering with the analysis of proteins and DNA. Ideally one would go from nanostructures up to much larger scale structures. Multiscale modeling refers to modeling in which multiple models at different scales of resolution are used to describe a system (Ibrahimbegovic and Papadrakakis, 2010). The development of advanced numerical procedures for multi-scale problems is a major challenge as many phenomena in structural engineering involve multiple scales and multi-scale methods are becoming the current trend in many branches of science providing major challenges in fields like nano-technology, fluid flows, bioengineering, material modeling and others.

\section{Multi-Physics Simulation}

To simulate nature, we need to consider multiple physical models or multiple simultaneous physical phenomena. Some of the most exciting challenges is the application of computational methods to problems of multi-physics nature, such as thermo-mechanical; electromagnetic-mechanical; thermo-chemical; fluid-structure interaction; bio-mechanics engineering; etc. Multi-physics methods can be also combined with multi-scale approaches, further increasing the complexity (Cross et al., 2007; de Borst, 2008).

\section{Modeling 3D Printing}

An admirable goal of 3D printing is the creation of material structures that are optimized to fit a particular structural application while at the same time minimizing the material weight and cost (shape or size optimization). 3D printing is still a new technology, particularly when it comes to the mechanical properties of 3D-printed specimens and structures (Killi and Morrison, 2016). 3D-printed parts differ from traditionally manufactured parts because of the weak adhesion of vertical layers, a wide variety of printing parameter variables and the behavior of the outer surface, which performs differently than the interior geometry. 3D printing challenges the ways that FEA methods are traditionally employed to assess the structural adequacy of engineered models with the complex internal geometries of 3D printed parts and components (Kazakis et al., 2017). The simulation of 3D printing is a coupled problem, a complex process that may involve not only structural strength but also thermal interactions, phase changes and others.

\section{Modeling of Uncertainties}

During the last decades, the engineering community acknowledged the importance of uncertainties on the performance of structures and engineering systems in general (Jensen and Iwan, 1992; Bulleit, 2008). Uncertainty quantification provides metrics to study the relationship between imprecisely prescribed model inputs and the model's predictions (Papadrakakis et al., 2005). However, the explicit quantification of uncertainties is still a major challenge (Takewaki, 2015). The development of appropriate methods for uncertainty quantification will receive much attention in the years to come with different computational models such as fuzzy analysis, classical probabilities, probabilistic hazard analysis (Mori et al., 2017), methods for uncertainty quantification such as robust design, reliability-based design (Lagaros et al., 2007), life-cycle optimal design (Mitropoulou and Lagaros, 2016), sensitivity analysis and others. These methods will create new opportunities to meet the long-standing challenge of delivering quantitative predictivity in computational mechanics and engineering.

\section{High-Performance and Cloud Computing}

High precision results for large-scale scientific problems often require tremendous computational power, an investment which is expensive and difficult to access. A solution to this problem can be found in high-performance and cloud computing. Although high-performance computing provides new and interesting opportunities to solve large-scale structural engineering problems (Papadrakakis et al., 2011; Hori et al., 2018), the development of new computational models and algorithms that exploit the unique architecture of these machines still remains a challenge (Adeli and Soegiarso, 1998). Cloud Computing is a model for enabling convenient, ondemand network access to a shared pool of configurable computing resources that can be rapidly provisioned and released with minimal management effort (Mell and Grance, 2011). An example of a high-performance cloud-based open-source framework is the new SimCenter (Computational Modeling and Simulation Center), a component of NSF-supported NHERI (Natural Hazards Engineering Research Infrastructure). The NHERI is a distributed, multi-user facility that provides the natural hazards engineering research and education community with access to research infrastructure. The goal of SimCenter is to provide the community with access to next generation opensource computational modeling and simulation software tools, 
allowing multidisciplinary specialists to collaborate on solutions to complex natural hazard engineering problems regardless of their local resources and geographic proximity.

\section{CONCLUSIONS}

Structural engineering has endured extraordinary challenges in recent years worldwide. Many of the tasks that a structural engineer used to do on his/her own in the past are now being done by computers. New ideas have evolved, outside the scope of prescriptive design codes and the use of computers is dominant nowadays. Very powerful capabilities are now available for the simulation and analysis of structures, yet there are still

\section{REFERENCES}

Adeli, H., and Soegiarso, R. (1998). High Performance Computing in Structural Engineering. Boca Raton, FL: CRC Press.

Babuška, I., and Melenk, J. M. (1997). The Partition of Unity Method. Int. J. Numer. Meth. Engng. 40, 727-758. doi: 10.1002/(SICI)10970207(19970228)40:4<727::AID-NME86>3.0.CO;2-N

Bathe, K.-J. (2003). "The key challenges in computational mechanics," in IACM Expressions, 10-12. Available online at: https://www.researchgate.net/ publication/237263439_The_Key_Challenges_in_Computational_Mechanics

Brebbia, C. A. (1980). The Boundary Element Method for Engineers, 2nd Edn, London, UK: Pentech Press Ltd.

Bulleit, W. M. (2008). Uncertainty in structural engineering. Pract. Period. Struct. Des. Constr. 13, 24-30. doi: 10.1061/(ASCE)1084-0680(2008)13:1(24)

Chen, J.-S., Hillman, M., and Chi, S.-W. (2017). Meshfree methods: progress made after 20 years. J. Engrg. Mech. 143, 04017001. doi: 10.1061/(ASCE)EM.1943-7889.0001176

Chi, S.-W., Chen, J.-S., Hu, H.-Y., and Yang, J. P. (2013). A gradient reproducing kernel collocation method for boundary value problems. Int. J. Numer. Meth. Engng. 93, 1381-1402. doi: 10.1002/nme.4432

Clough, R. W. (1960). "The finite element method in plane stress analysis," Proceedings of ASCE 2nd Conference on Electronic Computation (Pittsburgh, PA).

Coimbra, C. F. M. (2003). Mechanics with variable-order differential operators. Ann. Phys. 12, 692-703. doi: 10.1002/andp.200310032

Cross, M., Croft, T. N., Slone, A. K., Williams, A. J., Christakis, N., Patel, M. K., et al. (2007). Computational modelling of multi-physics and multiscale processes in parallel. Int. J. Comput. Methods Eng. Sci. Mech. 8, 63-74. doi: $10.1080 / 15502280601149510$

de Borst, R. (2008). Challenges in computational materials science: multiple scales, multi-physics and evolving discontinuities. Comput. Mater. Sci. 43, 1-15. doi: $10.1016 /$ j.commatsci.2007.07.022

Felippa, C. (2017). Introduction to Finite Element Methods (ASEN 5007). University of Colorado at Boulder.

Fredholm, I. (1903). Sur une classe d'equations fonctionnelles. Acta Math. 27, 365-390. doi: 10.1007/BF02421317

Gingold, R. A., and Monaghan, J. J. (1977). Smoothed particle hydrodynamics: theory and application to non-spherical stars. Mon. Not. R. Astron. Soc. 181, 375-389. doi: 10.1093/mnras/181.3.375

Hori, M., Ichimura, T., Wijerathne, L., Ohtani, H., Chen, J., Fujita, K., et al. (2018). Application of high performance computing to earthquake hazard and disaster estimation in urban area. Front. Built Environ. 4:1. doi: 10.3389/fbuil.2018.00001

Hughes, T. J. R., Cottrell, J. A., and Bazilevs, Y. (2005). Isogeometric analysis: CAD, finite elements, NURBS, exact geometry and mesh refinement. Comput. Methods Appl. Mech. Engrg. 194, 4135-4195. doi: 10.1016/j.cma.2004.10.008

Ibrahimbegovic, A., and Papadrakakis, M. (2010). Multi-scale models and mathematical aspects in solid and fluid mechanics. Comput. Methods Appl. Mech. Engrg. 199, 1241. doi: 10.1016/j.cma.2010.01.012 many exciting research challenges and the field of computational structural engineering will continue to grow and increasingly contribute to technological development. We strongly believe that we are only at the beginning of the use of computer simulations and we only now begin to understand the extent to which these will influence and enrich the engineering profession and our lives in general.

\section{AUTHOR CONTRIBUTIONS}

VP and GT: Contributed their thoughts and research experience to the conception and design of the work and drafting of the article.

Jaswon, M. A. (1963). Integral equation methods in potential theory. Proc. R. Soc. Lond. Ser. A. Math. Phys. Sci. 275, 23-32.

Jensen, H., and Iwan, W. D. (1992). Response of systems with uncertain parameters to stochastic excitation. J. Engrg. Mech., 118, 1012-1025. doi: 10.1061/(ASCE)0733-9399(1992)118:5(1012)

Kansa, E. J. (1990a). Multiquadrics-A scattered data approximation scheme with applications to computational fluid-dynamics-I surface approximations and partial derivative estimates. Comput. Math. Appl. 19, 127-145.

Kansa, E. J. (1990b). Multiquadrics-A scattered data approximation scheme with applications to computational fluid-dynamics-II solutions to parabolic, hyperbolic and elliptic partial differential equations. Comput. Math. Appl. 19, $147-161$.

Katsikadelis J. T. (1994). “The analog equation method - a powerful BEM-based solution technique for solving linear and nonlinear engineering problems," in Proceedings of the 16th International Boundary Element Method Conference (BEM XVI), July 12-15, ed C. A. Brebbia (Southampton: Computational Mechanics Publications), 167-182.

Katsikadelis, J. T. (2016). The Boundary Element Method for Engineers and Scientists: Theory and Applications, 2nd Edn. London, UK: Academic Press.

Katsikadelis, J. T. (2018). Numerical solution of variable order fractional differential equations. arXiv: 1802.00519v1 [math.NA].

Kazakis, G., Kanellopoulos, I., Sotiropoulos, S., and Lagaros, N. D. (2017) Topology optimization aided structural design: interpretation, computational aspects and 3D printing. Heliyon 3:e00431. doi: 10.1016/j.heliyon.2017.e00431

Killi, S., and Morrison, A. (2016). Fea and 3D printing, the perfect match? Int. J. Mech. Syst. Eng. 2:111. doi: 10.15344/2455-7412/2016/111

Lagaros, N. D., Plevris, V., and Papadrakakis, M. (2007). Reliability based robust design optimization of steel structures. Int. J. Simul. Multidisci. Des. Optim. 1, 19-29. doi: 10.1051/ijsmdo:2007003

Libersky, L. D., Petschek, A. G., Carney, T. C., Hipp, J. R., and Allahdadi, F. A. (1993). High strain lagrangian hydrodynamics: a three-dimensional SPH code for dynamic material response. J. Comput. Phys. 109, 67-75. doi: 10.1006/jcph.1993.1199

Liu, W. K., Jun, S., and Zhang, Y. F. (1995). Reproducing kernel particle methods. Int. J. Numeric. Methods Fluids 20, 1081-1106. doi: 10.1002/fld.1650200824

Lucy, L. B. (1977). A numerical approach to the testing of the fission hypothesis. Astron. J. 82, 1013-1024. doi: 10.1086/112164

Mazzoni, S., Mckenna, F., Scott, M. H., and Fenves, G. L. (2006). The Open System for Earthquake Engineering Simulation (OpenSEES) User Command-Language Manual. Berkeley, CA: CiteSeerX.

Mell, P., and Grance, T. (2011). The NIST Definition of Cloud Computing. NIST Special Publication 800-145, National Institute of Standards and Technology. doi: 10.6028/NIST.SP.800-145

Mitropoulou, C. C., and Lagaros, N. D. (2016). Life-cycle cost model and design optimization of base-isolated building structures. Front. Built Environ. 2:27. doi: 10.3389/fbuil.2016.00027

Moës, N., Dolbow, J., and Belytschko, T. (1999). A finite element method for crack growth without remeshing. Int. J. Numer. Meth. Engng. 46, 131-150. doi: 10. 1002/(SICI)1097-0207(19990910)46:1\&lt;131::AID-NME726\&gt;3.0.CO;2-J 
Mori, N., Muhammad, A., Goda, K., Yasuda, T., and Ruiz-Angulo, A. (2017). Probabilistic tsunami hazard analysis of the pacific coast of Mexico: case study based on the 1995 colima earthquake tsunami. Front. Built Environ. 3:34. doi: 10.3389/fbuil.2017.00034

Nature (2018). Computational Methods. Available online at: https://www.nature. com/subjects/computational-methods (Accessed March 4).

Papadrakakis, M., Lagaros, N. D., and Plevris, V. (2005). Design optimization of steel structures considering uncertainties. Eng. Struct. 27, 1408-1418. doi: 10.1016/j.engstruct.2005.04.002

Papadrakakis, M., Stavroulakis, G., and Karatarakis, A. (2011). A new era in scientific computing: domain decomposition methods in hybrid CPUGPU architectures. Comput. Methods Appl. Mech. Engrg. 200, 1490-1508. doi: 10.1016/j.cma.2011.01.013

Partridge, P. W., Brebbia, C. A., and Wrobel, L. C. (1991). Dual Reciprocity Boundary Element Method. Dordrecht: Springer

Reddy, J. N. (2005). An Introduction to the Finite Element Method, 3rd Edn. New York, NY: McGraw-Hill Education.
Sirca, G. F., and Adeli, H. (2012). System identification in structural engineering. Sci. Iranica 19, 1355-1364. doi: 10.1016/j.scient.2012.09.002

Symm, G. T. (1963). Integral equation methods in potential theory. Proc. R. Soc. Lond. Ser. A. Math. Phys. Sci. 275, 33-46.

Takewaki, I. (2015). Beyond uncertainties in earthquake structural engineering. Front. Built. Environ. 1:1. doi: 10.3389/fbuil.2015.00001

Conflict of Interest Statement: The authors declare that the research was conducted in the absence of any commercial or financial relationships that could be construed as a potential conflict of interest.

Copyright $\odot 2018$ Plevris and Tsiatas. This is an open-access article distributed under the terms of the Creative Commons Attribution License (CC BY). The use, distribution or reproduction in other forums is permitted, provided the original author(s) and the copyright owner are credited and that the original publication in this journal is cited, in accordance with accepted academic practice. No use, distribution or reproduction is permitted which does not comply with these terms. 